YITP-97-24

May 1997

\title{
Hamiltonian structures for compact homogeneous universes
}

\author{
Masayuki TANIMOTO周, TATSUHIKo KOIKE ${ }^{b}$ AND AKIo HOSOYA \\ $\dagger$ Yukawa Institute for Theoretical Physics, Kyoto University, Kyoto 606-01, Japan. \\ Electronic mail: tanimoto@yukawa.kyoto-u.ac.jp \\ b Department of Physics, Keio University, Kanagawa 230, Japan. \\ Electronic mail: koike@rk.phys.keio.ac.jp \\ 七 Department of Physics, Tokyo Institute of Technology, Tokyo 152, Japan. \\ Electronic mail: ahosoya@th.phys.titech.ac.jp
}

\begin{abstract}
Hamiltonian structures for spatially compact locally homogeneous vacuum universes are investigated, provided that the set of dynamical variables contains the Teichmüller parameters, parameterizing the purely global geometry. One of the key ingredients of our arguments is a suitable mathematical expression for quotient manifolds, where the universal cover metric carries all the degrees of freedom of geometrical variations, i.e., the covering group is fixed. We discuss general problems concerned with the use of this expression in the context of general relativity, and demonstrate the reduction of the Hamiltonians for some examples. For our models, all the dynamical degrees of freedom in Hamiltonian view are unambiguously interpretable as geometrical deformations, in contrast to the conventional open models.
\end{abstract}

*JSPS Research Fellow 


\section{INTRODUCTION}

Homogeneous cosmological models [1 3 have been served as a good arena for, e.g., quantum cosmology (e.g., [4.5]), as well as observational cosmology (e.g. [6]), after first investigated in connection with the singularity problem [7]. As for Hamiltonian structures of them, however, there are controversies. For example, it is well known that the models known as Bianchi class B do not possess a natural Hamiltonian reduced from the full Hamiltonian (See, e.g., Ref. [2], p.193). Even for the class A models, a sort of discrepancies of dynamical degrees of freedom is pointed out by Ashtekar and Samuel [8]. For example, the Kasner solution, which is the vacuum solution of Bianchi I, gives one for the number of dynamical degrees of freedom, i.e., the number of free parameters which can be specified freely at an initial Cauchy surface. Odd number of dynamical degrees of freedom, however, cannot come out from a Hamiltonian system. So, when a Hamiltonian is needful, people usually work with the diagonal model, which has three dynamical variables and gives four dynamical degrees of freedom in the Hamiltonian view. Moreover, if we work with the full, not diagonal model, which may be the most natural in the Hamiltonian view, we have ten dynamical degrees of freedom with six dynamical variables. Thus, three numbers (i.e., 1, 4 and 10) of dynamical degrees of freedom are possible for Bianchi I!

However, this discrepancy is for the conventional open model. It is a recent progress [9. 10] that a satisfactory framework has been established for the construction of spatially compact locally homogeneous spacetimes. (We shall hereafter refer to Refs. [9] and [10] as I and II, respectively.) We can now also unambiguously count the dynamical degrees of freedom in the above sense. In this framework, we "compactify" a usual spatially open homogeneous model by identifying spatial points appropriately. So, the new parameters come into the model which parameterize the identifications. An important point in dynamical view is that this compactification gives rise to new degrees of freedom of spatial deformations, known in mathematics as Teichmüller deformations [11, [12]. Teichmüller deformations obviously do not vary any local geometries. We call the parameters which parameterize the Teichmüller deformations Teichmüller parameters, and denote them collectively as $\boldsymbol{\tau}$. The natural dynamical variables of a spatially compact homogeneous ( $\mathrm{SCH}$ ) spacetime therefore consist of $(\boldsymbol{r}, \boldsymbol{\tau}, v)$, where $\boldsymbol{r}$ are the (local) curvature parameters, and $v$ is the total spatial volume. (For definiteness, we consider a vacuum case.) We denote the space spanned by $(\boldsymbol{r}, \boldsymbol{\tau}, v)$ and the cotangent bundle of it as $F$ and $P=T_{*} F$, respectively, and call $P$ the pseudo-phase space. What we shall investigate in this paper is whether $P$ admits a Hamiltonian structure, so in other words, whether it is good enough to call it a phase space.

We shall concentrate on the natural Hamiltonian structures, i.e., we take interests only in the phase spaces reduced from the full phase space which is made from the Einstein-

Hilbert action. Thus, all the dynamical parameters $(\boldsymbol{r}, \boldsymbol{\tau}, v)$ should appear as the metric 
components. Note that in our approach in II, parameters $(\boldsymbol{r}, \boldsymbol{\tau}, v)$ appeared both in the universal cover metric and the covering map — that is, the dynamical degrees of freedom were distributed in the two parts. To put them together into the universal cover metric, we need to be able to fix the covering map in an appropriate sense. In other words, all the dynamical degrees of freedom of the compact model need to be able to be expressed in the universal cover metric with a fixed covering map. Though at first sight this seems always possible, we should note that the metric obtained in this way, which has finite degrees of freedom of variation, does not always define a consistent cut in the full phase space. (This is the same situation as that for a partial differential equation, an arbitrary ansatz of solution does not lead to a true solution.) We will show for which models we can do this, and show why we cannot for the models we cannot.

The organization of this paper is as follows. In section प1], we review the previous work briefly, which gives a basis of our argument in the subsequent sections. In section III, we show how we can fix the covering map and thereby the universal cover metric can carry all the dynamical degrees of freedom. A class of diffeomorphisms (TDs) and a subclass of it (HPTDs) are introduced. Section $\mathbb{\square V}$ is devoted to explicit examples. Section $\square$ is for conclusions and remarks.

As we have already done, we abbreviate the words spatially compact homogeneous (or more precisely, spatially compact and spatially locally homogeneous) spacetimes as SCH spacetimes. Words in the title compact homogeneous universes are equivalent to the words $S C H$ spacetimes.

\section{CONSTRUCTION AND DYNAMICAL ASPECTS OF COMPACT HOMOGENEOUS UNIVERSES}

In this section, we briefly review the previous work, which concerns the construction of $\mathrm{SCH}$ spacetimes, and the dynamical aspects of them, namely the time-developments of the dynamical variables and the dynamical degrees of freedom. We refer the reader to II for explicit examples.

First, let us summarize the construction of the smooth set of compact homogeneous universes as a natural dynamical system. We assume that the topology of the compact homogeneous universes we consider is $M \times \mathbf{R}$, where $M$ is a spatial manifold and $\mathbf{R}$ corresponds to time. The fundamental group $\pi_{1}(M)$ of the space is supposed to be fixed in what follows.

Consider a spatially homogeneous spacetime $\left({ }^{(4)} \tilde{M}, \tilde{\mathrm{g}}_{a b}\right)$ and a homogeneous spatial section $\left(\tilde{M}_{t}, \tilde{h}_{a b}\right)$ of $\left({ }^{(4)} \tilde{M}, \tilde{\mathrm{g}}_{a b}\right)$. We denote the isometry groups of them as $\operatorname{Isom}^{(4)} \tilde{M}$ and $\operatorname{Isom} \tilde{M}_{t}$, respectively. [Here, the subscript $t$ parameterizes the spatial sections of $\left({ }^{(4)} \tilde{M}, \tilde{\mathrm{g}}_{a b}\right)$, but we only consider a fixed value of $t$ for a while, so that $t$ is just a reminder for $\tilde{M}_{t}$ being a section 
of ${ }^{(4)} \tilde{M}$.] Note that the subgroup of $\operatorname{Isom}^{(4)} \tilde{M}$ which preserves $\tilde{M}_{t}$ can be identified with a subgroup of Isom $\tilde{M}_{t}$. We call this subgroup the extendible isometry group of $\left(\tilde{M}_{t}, \tilde{h}_{a b}\right)$, and denote it as Esom $\tilde{M}_{t}$. To compactify $\left(\tilde{M}_{t}, \tilde{h}_{a b}\right)$, we embed $\pi_{1}(M)$ not into Isom $\tilde{M}_{t}$ but into Esom $\tilde{M}_{t}$, since by doing so, we can obtain a total smooth SCH spacetime $\left({ }^{(4)} M, \mathrm{~g}_{a b}\right)$. This is simply because Esom $\tilde{M}_{t}$ and therefore the embedding $\Gamma \subset$ Esom $\tilde{M}_{t}$ are also subgroups of Isom ${ }^{(4)} \tilde{M}$, so that the quotient $\left({ }^{(4)} \tilde{M}, \tilde{\mathrm{g}}_{a b}\right) / \Gamma=\left({ }^{(4)} M, \mathrm{~g}_{a b}\right)$ is guaranteed to be smooth.

This construction of a compact homogeneous universe tells us what define the natural smooth set of compact homogeneous universes as our dynamical system. We think they are the fundamental group of the spatial section $\pi_{1}(M)$ and the group structure of Esom $\tilde{M}_{t}$. We therefore fix the two groups, as well as an Einstein's equation, then think of the set $C$ of all possible compact homogeneous universes prescribed by them as a candidate of the natural smooth set. The set $C$ is, more explicitly, defined as the smooth set of all possible pairs $(u, \Gamma)$ of a universal covering spacetime $u$ and a covering group $\Gamma$ such that - (1) Each $u$ has a spatial section admitting the same extendible isometry group Esom $\tilde{M}_{t}$. (2) Each $u$ satisfies the same Einstein's equation. (3) Each $\Gamma$ acting on $u$ is a possible embedding of $\pi_{1}(M)$ into Esom $\tilde{M}_{t}$. We also define $U$ as the smooth set of universal covers which satisfy the conditions (1) and (2).

Each element $(u, \Gamma)$ in $C$ is naturally identified with the compact homogeneous universe $u / \Gamma$. Our natural smooth set $\bar{C}$ of compact homogeneous universes shall be obtained by identifying the isometric elements in $C$. To do this, we summarize key facts on diffeomorphisms on a covering space shortly.

Let $\left(\tilde{M}, \tilde{q}_{a b}\right)$ be an arbitrary homogeneous manifold, and $\left(\tilde{M}, \tilde{q}_{a b}\right) / \Gamma$ be its quotient. If we consider a diffeomorphism $\phi: \tilde{M} \rightarrow \tilde{M}$, then the following two quotients are manifestly isometric;

$$
\left(\tilde{M}, \tilde{q}_{a b}\right) / \Gamma \simeq\left(\tilde{M}, \phi_{*} \tilde{q}_{a b}\right) / \phi^{-1} \circ \Gamma \circ \phi,
$$

where $\phi_{*}$ is the "pullback" (i.e. the induced map) of $\phi$. (Since both $\Gamma$ and $\phi$ act on $\tilde{M}$, the composition of them in the right hand side is well-defined.) We can be benefited from this relation in two (or more) practical contexts - The first is just to simplify the universal cover metric $\tilde{q}_{a b}$, while the second is to deform the covering group $\Gamma$ suitably. Though we will utilize the second in the next section, our main focus in this section is in the first. Another importance comes when $\phi$ is an isometry of the universal cover $\left(\tilde{M}, \tilde{q}_{a b}\right)$, i.e., $\phi_{*} \tilde{q}_{a b}=\tilde{q}_{a b}$. In this case, it holds

$$
\left(\tilde{M}, \tilde{q}_{a b}\right) / \Gamma \simeq\left(\tilde{M}, \tilde{q}_{a b}\right) / \phi^{-1} \circ \Gamma \circ \phi .
$$

So, we can define an equivalence relation in the covering group by

$$
\Gamma \sim \phi^{-1} \circ \Gamma \circ \phi
$$


called conjugation. With this, we can simplify the covering group, even after we have exhausted diffeomorphisms on $\tilde{M}$ to simplify $\tilde{q}_{a b}$.

Now, the subsequent procedure to get $\bar{C}$ is almost straightforward. Introducing the equivalence relation by diffeomorphism in $U$, we obtain a smooth set $\bar{U}$ of representative elements. For convenience, we often identify $\bar{U}$ with a universal covering spacetime with $n=\operatorname{dim} \bar{U}$ smooth parameters, and denote these parameters and the (parameteric) universal cover as $\mathbf{u}$ and $\left({ }^{(4)} \tilde{M}, \tilde{\mathrm{g}}_{a b}[\mathbf{u}]\right)$, respectively.

For a fixed $u \in \bar{U}$, we give all the possible $\Gamma$ 's, and denote the smooth set of the pairs, $(u, \Gamma)$ 's, as $C_{u}$. Then, we construct the conjugacy class $\bar{C}_{u}$ of $C_{u}$ by $\operatorname{Esom} \tilde{M}_{t}$, i.e., equivalence class defined by $(u, \Gamma) \sim\left(u, a \circ \Gamma \circ a^{-1}\right)$, where $a \in \operatorname{Esom} \tilde{M}_{t}$. We finally consider the smooth set $\bar{C}$ of spacetimes obtained from $\bar{C}_{u}$ with varying $u$, i.e., $\bar{C} \equiv\left\{c \mid c \in \bar{C}_{u}, u \in \bar{U}\right\}$. We think of $\bar{C}$ as the natural dynamical system we wanted, consisting of compact homogeneous universes. Like $\bar{U}$, we identify $\bar{C}$ with a compact homogeneous universe with $n+m$ smooth parameters, where $m=\operatorname{dim} \bar{C}_{u}$. We denote the $m$ parameters in $\bar{C}_{u}$ and the (parameteric) compact homogeneous universe as, respectively, $\mathbf{g}$ and $\left({ }^{(4)} \tilde{M}, \tilde{\mathrm{g}}_{a b}[\mathbf{u}]\right) / \Gamma_{\mathbf{g}}$. The number of dynamical degrees of freedom is now simply given by $\operatorname{dim} \bar{C}=\operatorname{dim} \bar{U}+\operatorname{dim} \bar{C}_{u}=n+m$. This completes our construction.

For example, when the extendible isometry group is given by a Bianchi group, we can take, without loss of generality, the universal cover metric as

$$
\mathrm{d} s^{2}=-\mathrm{d} t^{2}+h_{\mu \nu}(t) \sigma^{\mu} \sigma^{\nu}
$$

where $\sigma^{\mu}$ are the invariant 1-forms of the Bianchi group, and $h_{\mu \nu}$ is a nondegenerate symmetric $3 \times 3$ matrix function of $t$. We substitute this into Einstein's equation, and then subtract the degrees of freedom of diffeomorphisms from the solution obtained. We usually do this last step with the homogeneity preserving diffeomorphisms (HPDs) [8], which are defined by the condition that they induce "rotations" among the invariant 1-forms, i.e., for an HPD $\eta$, it holds;

$$
\eta_{*}: \sigma^{\mu} \rightarrow f^{\mu}{ }_{\nu} \sigma^{\nu}
$$

where $f^{\mu}{ }_{\nu}$ is a constant $3 \times 3$ matrix. (Possible $f^{\mu}{ }_{\nu}$ 's comprise a subgroup of GL(3).) The final form of the solution takes, in many cases, a diagonal form;

$$
\mathrm{d} s^{2}=-\mathrm{d} t^{2}+h_{11}(t ; \mathbf{u})\left(\sigma^{1}\right)^{2}+h_{22}(t ; \mathbf{u})\left(\sigma^{2}\right)^{2}+h_{33}(t ; \mathbf{u})\left(\sigma^{3}\right)^{2} .
$$

This gives the set $\bar{U}$, or the metric $\tilde{\mathrm{g}}_{a b}[\mathbf{u}]$. (The explicit form of $h_{\mu \nu}(t ; \mathbf{u})$ depends, of course, upon the given Bianchi group.) $\Gamma_{\mathbf{g}}$ is obtained by exhausting possible conjugations with respect to the extendible isometries, in the possible embeddings of the fundamental group $\pi_{1}(M)$ into the extendible isometry group. 
We note that the dynamical variables in configuration space are defined with respect to the intrinsic geometry of the spatial section. Hence, like that it was important to subtract the degrees of freedom of diffeomorphisms and conjugations of the spacetime when determining the number of dynamical degrees of freedom, it is important to subtract the degrees of freedom of diffeomorphisms and conjugations of the space to determine the values of the dynamical variables. This process is basically the same as what has been presented for spacetime, except for considering Isom $\tilde{M}_{t}$ instead of Esom $\tilde{M}_{t}$. In I, we have presented parameterizations for almost all compact locally homogeneous three-manifolds — Each parameterization is like

$$
\left(\tilde{M}, \tilde{h}_{a b}^{\text {std }}[\boldsymbol{r}]\right) / A_{\boldsymbol{\tau}},
$$

where the standard universal cover $\left(\tilde{M}, \tilde{h}_{a b}^{\text {std }}[\boldsymbol{r}]\right)$ is parameterized so as to contain no degrees of freedom of diffeomorphisms on $\tilde{M}$, and the covering group $A_{\boldsymbol{\tau}}$ acting on $\left(\tilde{M}, \tilde{h}_{a b}^{\text {std }}[\boldsymbol{r}]\right)$ is parameterized so as to be free from the conjugations with respect to the (intrinsic) isometry group $\operatorname{Isom} \tilde{M} \simeq \operatorname{Isom} \tilde{M}_{t}$. As in II, we call the parameters $(\boldsymbol{r}, \boldsymbol{\tau})$ the dynamical variables. Each compact locally homogeneous spatial section $\left(\tilde{M}_{t}, \tilde{h}_{a b}[\mathbf{u}]\right) / \Gamma_{\mathbf{g}}$ of the solution $\left({ }^{(4)} \tilde{M}, \tilde{\mathrm{g}}_{a b}[\mathbf{u}]\right) / \Gamma_{\mathbf{g}}$ is isometric to $\left(\tilde{M}, \tilde{h}_{a b}^{\text {std }}[\boldsymbol{r}]\right) / A_{\boldsymbol{\tau}}$ for some $\boldsymbol{r}$ and $\boldsymbol{\tau}$. The dynamical variables $(\boldsymbol{r}, \boldsymbol{\tau})$ can therefore be identified with functions of time $\boldsymbol{r}=\boldsymbol{r}(t ; \mathbf{u})$, and $\boldsymbol{\tau}=\boldsymbol{\tau}(t ; \mathbf{u}, \mathbf{g})$, containing $(\mathbf{u}, \mathbf{g})$ as constant parameters. $(\boldsymbol{r}$ is determined only from the universal cover metric $\tilde{h}_{a b}[\mathbf{u}]$, so that $\boldsymbol{r}$ is independent of $\mathbf{g}$.) When considering a Bianchi group as the extendible isometry group, $\boldsymbol{r}$ and $\boldsymbol{\tau}$ depend on $t$ and $\mathbf{u}$ through the component functions $\mathbf{h} \equiv\left(h_{11}(t ; \mathbf{u}), h_{22}(t ; \mathbf{u}), h_{33}(t ; \mathbf{u})\right)$ in Eq.(6), so that we may write as $\boldsymbol{r}=\boldsymbol{r}(\mathbf{h})$, and $\boldsymbol{\tau}=\boldsymbol{\tau}(\mathbf{h}, \mathbf{g})$. We executed this procedure for some explicit models in II.

For convenience, we often reparametrize $\tilde{h}_{a b}^{\text {std }}[\boldsymbol{r}]$ or $A_{\boldsymbol{\tau}}$, and factor out $v$ to parameterize the volume. In such a case, the dynamical variables are $(\boldsymbol{r}, \boldsymbol{\tau}, v)$, and they are functions of time containing $(\mathbf{u}, \mathbf{g})$ as constant parameters. We shall refer to the steps here of finding the time-development of the dynamical variables as the spacetime approach, in distinction from the dynamical approach which will be presented in the next section.

\section{FIXING COVERING MAPS AND THE NATURAL HAMILTONIAN STRUCTURES}

As is well known, general relativity possesses Hamiltonian formalism (e.g. [3]). In this section, we consider how we can obtain, from the full phase space, a reduced phase space for our dynamical system of compact homogeneous universe. In our mathematical representation of a compact homogeneous universe in the previous section, part of dynamical degrees of freedom was contained in the covering map, which cannot be a canonical dynamical variable 
in ordinary way. In some cases, however, we can move to another representation in which the covering map contains no dynamical degrees of freedom and the universal cover metric carry all the ones. In these cases we may obtain the natural reduced Hamiltonian.

We first consider a reparametrization of compact locally homogeneous 3-manifolds. Remember that our standard parameterization of compact locally homogeneous 3-manifolds is like Eq.(7). (For simplicity, we do not factor out the total volume $v$ in this section.) This parameterization is of particular importance in that it is the most natural one among those of containing no redundant parameters. However, once we find such a parameteriza-

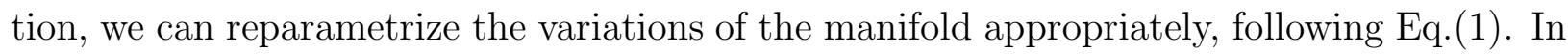
particular, we can fix the covering map, using a diffeomorphism $\phi_{\boldsymbol{\tau}}: \tilde{M} \rightarrow \tilde{M}$ such that

$$
A_{\boldsymbol{\tau}}=\phi_{\boldsymbol{\tau}} \circ A_{0} \circ \phi_{\boldsymbol{\tau}}^{-1},
$$

where $A_{0}$ is the covering group for a set of fixed Teichmüller parameters $\boldsymbol{\tau}=\boldsymbol{\tau}_{0}$. With this diffeomorphism, we obtain parameterization

$$
\left(\tilde{M}, \tilde{h}_{a b}^{\mathrm{dyn}}[\boldsymbol{r}, \boldsymbol{\tau}]\right) / A_{0},
$$

where

$$
\tilde{h}_{a b}^{\mathrm{dyn}}[\boldsymbol{r}, \boldsymbol{\tau}] \equiv \phi_{\boldsymbol{\tau} *} \tilde{h}_{a b}^{\mathrm{std}}[\boldsymbol{r}] .
$$

It is worth noting that $\phi_{\boldsymbol{\tau}}$ is a diffeomorphism which maps a fundamental region of the projection map $\pi_{0}$ of $A_{0}$ to a fundamental region of $\pi_{\boldsymbol{\tau}}$ of $A_{\boldsymbol{\tau}}$. We shall refer to $\phi_{\boldsymbol{\tau}}$ as a Teichmüller diffeomorphism (TD).

We may be able to see the metric (10) as a "dynamical" metric. That is, we expect there exists a spacetime metric $\tilde{g}_{a b}^{\text {dyn }}(\boldsymbol{r}, \boldsymbol{\tau})$ whose spatial part is given by Eq.(10), where $\boldsymbol{r}$ and $\boldsymbol{\tau}$ are functions of time $t$. If we start with such a "dynamical" spacetime metric with $\boldsymbol{r}(t)$ and $\boldsymbol{\tau}(t)$ being free functions of $t$, then we would be able to find the time-development of them directly from Einstein's equation. (Note that by definition, the quotient of the solution obtained in this way is free from the degrees of freedom of diffeomorphisms.) This may give another approach, which we call the dynamical approach, to obtain the time-development of the dynamical variables $(\boldsymbol{r}(t), \boldsymbol{\tau}(t))$. This is similar to the treatment employed in $(2+1)$-gravity [13].

We notice, however, that TDs are not unique, so that many possibilities of different parameterizations of Eq.(91) exist. Moreover, even for a given parameterization of Eq.(9), there seem to exist many possibilities of ways of taking the dynamical spacetime metric, i.e., of taking the shift vector. (The spatial dependence of the lapse function results in an undesirable change of spatial foliation, but just $t$-dependence of the lapse function simply results in a reparametrization of $t$, which is not essential for our purpose. We therefore fix the 
lapse function to unity.) However, the ambiguity of the shift vector is not essential. What is essential is the choice of TD. To see this, suppose we are given the solution constructed unambiguously in the spacetime approach for given Esom $\tilde{M}_{t}$ and $\pi_{1}(M)$.

For an SCH spacetime to yield $\left(\tilde{M}_{t}, \tilde{h}_{a b}^{\text {dyn }}[\boldsymbol{r}, \boldsymbol{\tau}]\right) / A_{0}$ as its spatial part, this SCH spacetime need be of the form $\left({ }^{(4)} \tilde{M}, \tilde{\mathrm{g}}_{a b}^{\mathrm{dyn}}(\boldsymbol{r}, \boldsymbol{\tau})\right) / A_{0}$ with $A_{0}$ being a discrete subgroup of the extendible isometry group. Moreover, this $\mathrm{SCH}$ spacetime must be isometric to the SCH spacetime constructed in the spacetime approach, $\left({ }^{(4)} \tilde{M}, \tilde{\mathrm{g}}_{a b}[\mathbf{u}]\right) / \Gamma_{\mathbf{g}}$. Hence, we must have the following commutative diagram;

$$
\begin{array}{ccc}
\left({ }^{(4)} \tilde{M}, \tilde{\mathrm{g}}_{a b}[\mathbf{u}](t)\right) / \Gamma_{\mathbf{g}} & \stackrel{\psi_{\mathbf{g}}}{\longleftarrow} & \left({ }^{(4)} \tilde{M}, \tilde{\mathrm{g}}_{a b}^{\mathrm{dyn}}(\boldsymbol{r}(t), \boldsymbol{\tau}(t))\right) / A_{0} \\
\downarrow & \downarrow & \downarrow \\
\left(\tilde{M}_{t}, \tilde{h}_{a b}[t, \mathbf{u}]\right) / \Gamma_{\mathbf{g}} & \leftarrow\left(\tilde{M}, \tilde{h}_{a b}^{\mathrm{std}}[\boldsymbol{r}(t)]\right) / A_{\boldsymbol{\tau}(t)} \stackrel{\phi_{\boldsymbol{\tau}}}{\leftarrow} & \left(\tilde{M}_{t}, \tilde{h}_{a b}^{\mathrm{dyn}}[\boldsymbol{r}(t), \boldsymbol{\tau}(t)]\right) / A_{0}
\end{array} .
$$

Here, the vertical arrows stand for restricting to spatial manifolds, the horizontal arrows for mapping by diffeomorphisms, and we have inserted $t$-dependence explicitly. [We are consistently using square brackets [] to denote the parametric dependence of the arguments, while using parentheses ( ) to denote the usual dependence of the arguments.]

Let $\psi_{\mathbf{g}}$ be the diffeomorphism $\psi_{\mathbf{g}}:{ }^{(4)} \tilde{M} \rightarrow{ }^{(4)} \tilde{M}$ which connects the two spacetime manifolds in Eq.(11). That is, $\psi_{\mathbf{g}}$ is a diffeomorphism such that

$$
\Gamma_{\mathbf{g}}=\psi_{\mathbf{g}} \circ A_{0} \circ \psi_{\mathbf{g}}^{-1}
$$

Also, $\psi_{\mathbf{g}}$ preserves each spatial section (i.e., preserves the spatial foliation). With $\psi_{\mathbf{g}}$, the two universal cover metrics relate through

$$
\tilde{\mathrm{g}}_{a b}^{\mathrm{dyn}}(\boldsymbol{r}, \boldsymbol{\tau})=\psi_{\mathbf{g} * \tilde{\mathrm{g}}_{a b}}[\mathbf{u}]
$$

Note that the diffeomorphism which connects the left two spatial manifolds in Eq.(11) is uniquely specified. For example, when the extendible isometry group is given by a Bianchi group, it was the composition, $\eta \circ \iota: \tilde{M} \rightarrow \tilde{M}_{t}$, of an HPD $\eta$, used to simplify the universal cover metric to the standard metric, and an isometry $\iota$, corresponding to the conjugation. This, in turn, implies that the TD $\phi_{\boldsymbol{\tau}}$ and $\psi_{\mathbf{g}}$ are in one-to-one correspondence;

$$
\psi_{\mathbf{g}}=\eta \circ \iota \circ \phi_{\boldsymbol{\tau}}
$$

(Since $\psi_{\mathbf{g}}$ preserves the spatial foliation, $\psi_{\mathbf{g}}$ can be naturally identified with spatial diffeomorphisms. Conversely, since $\eta, \iota$, and $\phi_{\boldsymbol{\tau}}$ contain $t$ as parameter, they can be naturally identified with diffeomorphisms on the spacetime manifold. Eq.(14) is therefore well-defined on both $\tilde{M}_{t}$ and ${ }^{(4)} \tilde{M}$. Note, however, that $\iota$ is not in general an isometry when acting on the spacetime $\left({ }^{(4)} \tilde{M}, \tilde{g}_{a b}\right)$.) Thus, we can focus on $\psi_{\mathbf{g}}$ 's, instead of thinking of TDs. We may always find many varieties of foliation preserving diffeomorphisms $\psi_{\mathbf{g}}$ 's which satisfy 
Eq.(12), so that there still seem to exist many varieties of dynamical spacetime metrics. Note, however, that for arbitrary choices of $\psi_{\mathbf{g}}$, the right hand side of Eq.(13) would depend freely on $t, \mathbf{u}$ and $\mathbf{g}$;

$$
\psi_{\mathbf{g} * \tilde{g}_{a b}}[\mathbf{u}](t)=\left(\psi_{\mathbf{g} * \tilde{g}_{a b}}\right)[\mathbf{u}, \mathbf{g}](t) .
$$

Since we cannot, in general, "invert" the functions $\boldsymbol{r}=\boldsymbol{r}(t ; \mathbf{u})$, and $\boldsymbol{\tau}=\boldsymbol{\tau}(t ; \mathbf{u}, \mathbf{g})$, i.e., we cannot find $t=t(\boldsymbol{r}, \boldsymbol{\tau}), \mathbf{u}=\mathbf{u}(\boldsymbol{r}, \boldsymbol{\tau})$, and $\mathbf{g}=\mathbf{g}(\boldsymbol{r}, \boldsymbol{\tau})$, we would not be able to have the right dynamical metric as a functional of $\boldsymbol{r}(t)$ and $\boldsymbol{\tau}(t)$;

$$
\left(\psi_{\left.\mathbf{g} * \tilde{\mathrm{g}}_{a b}\right)}[\mathbf{u}, \mathbf{g}](t) \neq \tilde{\mathrm{g}}_{a b}^{\mathrm{dyn}}(\boldsymbol{r}(t), \boldsymbol{\tau}(t)) .\right.
$$

Obviously, to have the right dynamical metric, the pullback of $\tilde{\mathrm{g}}_{a b}[\mathbf{u}]$ by $\psi_{\mathbf{g}}$ must take a special form where $t, \mathbf{u}$, and $\mathbf{g}$ appears as algebraic combinations of $\boldsymbol{r}=\boldsymbol{r}(t ; \mathbf{u})$ and $\boldsymbol{\tau}=\boldsymbol{\tau}(t ; \mathbf{u}, \mathbf{g})$. This shows the origin of the fact that an arbitrary choice of TD in the dynamical approach leads us to only a bad ansatz for the solution of the field equation, no matter how we choose the shift vector. Conversely, if we choose a right TD, the right dynamical metric is unique due to a correspondence such as Eq.(14).

In general it is not trivial to find right TDs. Even worse, in some cases they may not exist. However, in special cases shown below the existence of the right TDs is guaranteed and we can carry out the dynamical approach.

Suppose the extendible isometry group is given by a Bianchi group $G$, and consider a class of SCH spacetimes such that we can find the TDs in the HPDs. We call the TDs implemented in (a subgroup of) the HPDs the homogeneity preserving Teichmüller diffeomorphisms (HPTDs). The dynamical metric (with zero shift vector) with the HPTDs becomes of the form (困), which is expected to consistently describe the time-development of the dynamical variables, since it is just an ordinary, but non-diagonal generally, Bianchi type spacetime metric. Esom $\tilde{M}_{t}=G$ is also satisfied. (One might still care about that possible momentum constraints would excessively reduce the number of dynamical degrees of freedom, but this is not the case. The momentum constraints correspond to the inner automorphisms, $\operatorname{Inn}(G)$, of the Bianchi group $G$ [E], which in turn correspond to the conjugations in the quotient space. Since we have so defined the TDs that no conjugations are contained in the quotient space, the dynamical metric will be free from the momentum constraints.) Thus, the HPTDs will give a right dynamical metric. This constitutes an essential ingredient of our actual reduction of the Hamiltonian.

Finally, we can easily find a necessary condition to have the HPTDs, which is given by

$$
\chi \geq \operatorname{dim} \bar{C}_{u},
$$

where $\operatorname{dim} \bar{C}_{u}$ equals the number of the parameters $\mathbf{g}$, and 


$$
\chi \equiv \operatorname{dim} \operatorname{Out}(G)=\operatorname{dim} \operatorname{HPDs}-\operatorname{dim} \operatorname{Inn}(G) .
$$

Here, $\operatorname{Out}(G)$ is the outer automorphism group of $G$, and we have used the fact that the HPDs comprise the automorphism group of $G$. The condition (17) can be understood from the observation that the $\psi_{\mathbf{g}}$ must be (time-independent) HPDs, modulo the gauge orbits generated by the momentum constraints.

In the next section, we demonstrate the dynamical approach and show the Hamiltonians by means of the HPTDs.

\section{FOUR EXAMPLES}

We in this section apply our argument to the four vacuum models investigated previously in II, namely the $\mathrm{b} / 1, \mathrm{f} 1 / 1(n)$, a1/1, and a2/1 models. The definitions of the four compact homogeneous universes are summarized in the table below, where the extendible isometry groups and the fundamental groups of the spatial sections are given. We have abbreviated the Bianchi N group as "BN". Explicit representations of $\pi_{1}\left(M_{\mathrm{b} / 1}\right)$ and $\pi_{1}\left(M_{\mathrm{f} 1 / 1(n)}\right)$ are presented in, respectively, Eqs.(14) II and (37) II. (For simplicity, we in this section refer to equations in I and II as $(1)_{\mathrm{I}},(1)_{\mathrm{II}}$, etc..)

\begin{tabular}{|c|c|c|c|c|}
\hline Model & Esom $\tilde{M}_{t}$ & Multiplication & rule for $\operatorname{Esom} \tilde{M}_{t}$ & $\pi_{1}$ \\
\hline $\mathrm{b} / 1$ & BII & $\left(\begin{array}{l}g^{1} \\
g^{2} \\
g^{3}\end{array}\right)\left(\begin{array}{l}h^{1} \\
h^{2} \\
h^{3}\end{array}\right)=$ & $\left(\begin{array}{c}g^{1}+h^{1} \\
g^{2}+h^{2} \\
g^{3}+h^{3}+g^{1} h^{2}\end{array}\right.$ & $\pi_{1}\left(M_{\mathrm{b} / 1}\right)$ \\
\hline $\mathrm{f} 1 / 1(n)$ & $\mathrm{BVI}_{0}$ & $\left(\begin{array}{l}g^{1} \\
g^{2} \\
g^{3}\end{array}\right)\left(\begin{array}{l}h^{1} \\
h^{2} \\
h^{3}\end{array}\right)$ & $=\left(\begin{array}{c}g^{1}+e^{-g^{3}} h^{1} \\
g^{2}+e^{g^{3}} h^{2} \\
g^{3}+h^{3}\end{array}\right)$ & $\pi_{1}\left(M_{\mathrm{f} 1 / 1(\mathrm{n})}\right)$ \\
\hline a1/1 & $\mathrm{BVII}_{0}$ & $\left(\begin{array}{l}g^{1} \\
g^{2} \\
g^{3}\end{array}\right)\left(\begin{array}{l}h^{1} \\
h^{2} \\
h^{3}\end{array}\right)=$ & 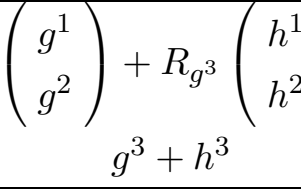 & $\pi_{1}\left(T^{3}\right)$ \\
\hline $\mathrm{a} 2 / 1$ & BI & $\left(\begin{array}{l}g^{1} \\
g^{2} \\
g^{3}\end{array}\right)\left(\begin{array}{l}h^{1} \\
h^{2} \\
h^{3}\end{array}\right.$ & $=\left(\begin{array}{c}g^{1}+h^{1} \\
g^{2}+h^{2} \\
g^{3}+h^{3}\end{array}\right)$ & $\pi_{1}\left(T^{3}\right)$ \\
\hline
\end{tabular}

In this table, $R_{g^{3}}$ is the rotation matrix by angle $g^{3}$, and the usual multiplication rule for matrix is understood there. The multiplication rules in this table will be applied without any notice in the subsequent subsections.

We will see that the HPTDs are found, the dynamical metrics can be written, and thereby the Hamiltonians are obtained, for the b/1, f1/1(n), and a2/1 models. For these models, 
$\chi=\operatorname{dim} \bar{C}_{u}$ holds, so that the condition (17) is satisfied. The a1/1 model does not satisfy this condition (but $0<\chi<\operatorname{dim} \bar{C}_{u}$ ). Nevertheless, we will succeed to have a Hamiltonian also for this model, if we admit a "degeneracy" of the dynamical variables. (See Sec.IVQ.)

All the arguments in the following subsections are basically parallel to the first one, Sec IVA.

\section{A. The b/1 model}

We begin with preparing the HPDs $\eta$ of Bianchi II, which are obtained from the invariance under Eq.(5) of the Maurer-Cartan relation

$$
\mathrm{d} \sigma^{1}=0, \quad \mathrm{~d} \sigma^{2}=0, \quad \mathrm{~d} \sigma^{3}=-\sigma^{1} \wedge \sigma^{2} .
$$

The invariant 1-forms $\sigma^{i}$ here of Bianchi II are defined by

$$
\sigma^{1} \equiv \mathrm{d} x, \sigma^{2} \equiv \mathrm{d} y, \sigma^{3} \equiv \mathrm{d} z-x \mathrm{~d} y
$$

using a local coordinate basis $(x, y, z)$. We find

$$
f_{3}^{1}=f_{3}^{2}=0, \quad f^{3}{ }_{3}=\bar{f}_{3}^{3} \equiv f_{1}^{1} f_{2}^{2}-f^{1}{ }_{2} f^{2}{ }_{1},
$$

and so that

$$
\eta_{*}:\left(\begin{array}{c}
\sigma^{1} \\
\sigma^{2} \\
\sigma^{3}
\end{array}\right) \rightarrow\left(\begin{array}{ccc}
f^{1}{ }_{1} & f^{1}{ }_{2} & 0 \\
f^{2}{ }_{1} & f^{2}{ }_{2} & 0 \\
f^{3}{ }_{1} & f^{3}{ }_{2} & \bar{f}^{3}{ }_{3}
\end{array}\right)\left(\begin{array}{c}
\sigma^{1} \\
\sigma^{2} \\
\sigma^{3}
\end{array}\right) .
$$

Moreover, by integration we have

$$
\eta:\left(\begin{array}{c}
x \\
y \\
z
\end{array}\right) \rightarrow\left(\begin{array}{c}
f^{1}{ }_{1} x+f^{1}{ }_{2} y \\
f^{2}{ }_{1} x+f^{2}{ }_{2} y \\
(1 / 2)\left(f^{1}{ }_{1} f^{2}{ }_{1} x^{2}+f^{1}{ }_{2} f_{2}{ }_{2} y^{2}\right)+f^{1}{ }_{2} f^{2}{ }_{1} x y+\bar{f}^{3}{ }_{3} z+f^{3}{ }_{1} x+f^{3}{ }_{2} y
\end{array}\right)
$$

We have set the integral constants, corresponding to isometries, zero. This is the general form of HPDs, up to isometry. There are six free parameters in it, which are constant with respect to the spatial coordinates $(x, y, z)$, but possibly depend on time $t$, depending upon the context.

Our standard universal cover metric, up to constant conformal factor, is (cf. Eq.(75) I)

$$
\mathrm{d} l^{2}=\left(\sigma^{1}\right)^{2}+\left(\sigma^{2}\right)^{2}+\left(\sigma^{3}\right)^{2}
$$

and the parameterization of the Teichmüller space is given by the following generators acting on the standard universal cover (cf. Eq.(129)I); 


$$
A_{\boldsymbol{\tau}}=\left\{\left(\begin{array}{c}
a_{1}{ }^{1} \\
0 \\
0
\end{array}\right),\left(\begin{array}{c}
a_{2}{ }^{1} \\
a_{2}{ }^{2} \\
0
\end{array}\right),\left(\begin{array}{c}
0 \\
0 \\
a_{1}{ }^{1} a_{2}{ }^{2}
\end{array}\right)\right\} .
$$

So, there are no universal cover parameters, and three Teichmüller parameters;

$$
\boldsymbol{r}=\emptyset, \quad \boldsymbol{\tau}=\left(a_{1}{ }^{1}, a_{2}{ }^{1}, a_{2}{ }^{2}\right) .
$$

We take $\boldsymbol{\tau}_{0}=(1,0,1)$, for later convenience. The dynamical variables are $\boldsymbol{\tau}$ and the volume $v$, which has been omitted from the standard universal cover metric basically for simplicity, but will come into the dynamical metric finally.

We can choose the unit cube (Fig.1) in $0 \leq x \leq 1,0 \leq y \leq 1$ and $0 \leq z \leq 1$ as a fundamental region of $\pi_{0}$. If we choose a fundamental region of $\pi_{\tau}$ such that the projection onto the $x-y$ plane is a parallelogram shown in Fig.2, then the HPTDs are determined by the requirement that they comprise a subgroup of HPDs. In fact, for the $x-y$ plane, TD $\phi_{\tau}$ must be the linear transformation

$$
\phi_{\boldsymbol{\tau}}:\left(\begin{array}{l}
x \\
y
\end{array}\right) \rightarrow\left(\begin{array}{c}
a_{1}{ }^{1} x+a_{2}{ }^{1} y \\
a_{2}{ }^{2} y
\end{array}\right),
$$

so that we have

$$
\phi_{\boldsymbol{\tau} *}:\left(\begin{array}{c}
\sigma^{1} \\
\sigma^{2}
\end{array}\right) \rightarrow\left(\begin{array}{cc}
a_{1}^{1} & a_{2}{ }^{1} \\
0 & a_{2}^{2}
\end{array}\right)\left(\begin{array}{l}
\sigma^{1} \\
\sigma^{2}
\end{array}\right) .
$$

This in turn implies (cf. Eq.(22))

$$
\phi_{\boldsymbol{\tau} *}:\left(\begin{array}{c}
\sigma^{1} \\
\sigma^{2} \\
\sigma^{3}
\end{array}\right) \rightarrow\left(\begin{array}{ccc}
a_{1}{ }^{1} & a_{2}{ }^{1} & 0 \\
0 & a_{2}{ }^{2} & 0 \\
0 & 0 & a_{1}{ }^{1} a_{2}{ }^{2}
\end{array}\right)\left(\begin{array}{c}
\sigma^{1} \\
\sigma^{2} \\
\sigma^{3}
\end{array}\right) .
$$

It is easy to observe that the TDs of Eq.(29) certainly comprise a subgroup of HPDs. Comparing with Eq.(23), we finally have

$$
\phi_{\boldsymbol{\tau}}:\left(\begin{array}{c}
x \\
y \\
z
\end{array}\right) \rightarrow\left(\begin{array}{c}
a_{1}{ }^{1} x+a_{2}{ }^{1} y \\
a_{2}{ }^{2} y \\
\frac{1}{2} a_{2}{ }^{1} a_{2}{ }^{2} y^{2}+a_{1}{ }^{1} a_{2}{ }^{2} z
\end{array}\right) .
$$

The image of the unit cube by this "HPTD" must be a fundamental region of $\pi_{\tau}$ (Fig.1), i.e., must satisfy Eq.(\$). In fact, for the generators of $A_{0}$

$$
A_{0}=\left\{\left(\begin{array}{l}
1 \\
0 \\
0
\end{array}\right),\left(\begin{array}{l}
0 \\
1 \\
0
\end{array}\right),\left(\begin{array}{l}
0 \\
0 \\
1
\end{array}\right)\right\}
$$


we can calculate

$$
\phi_{\boldsymbol{\tau}} \circ A_{0} \circ \phi_{\boldsymbol{\tau}}^{-1}=\left\{\left(\begin{array}{c}
a_{1}{ }^{1} \\
0 \\
0
\end{array}\right),\left(\begin{array}{c}
a_{2}{ }^{1} \\
a_{2}{ }^{2} \\
\frac{1}{2} a_{2}{ }^{1} a_{2}{ }^{2}
\end{array}\right),\left(\begin{array}{c}
0 \\
0 \\
a_{1}{ }^{1} a_{2}{ }^{2}
\end{array}\right)\right\},
$$

which certainly coincides with $A_{\tau}$, up to conjugation. (The third component of the second generator of Eq.(32) differs from that of Eq.(25), but this is not essential for our purpose, since we can make it zero by using an isometry $\iota$, i.e., $\phi_{\boldsymbol{\tau}} \rightarrow \phi_{\boldsymbol{\tau}} \circ \iota$ in Eq.(32). This change does not affect the form of the dynamical metric.) Thus, Eq.(30) is the right HPTDs.

The induced metric of Eq.(24) by $\phi_{\boldsymbol{\tau}}$ is obtained from the direct substitution of Eq.(29). Normalizing the induced metric to give $v^{2}$ as determinant, (and attaching " $-\mathrm{d} t^{2}$ ",) we obtain the dynamical metric

$$
\mathrm{d} s^{2}=-\mathrm{d} t^{2}+H_{\alpha \beta} \sigma^{\alpha} \sigma^{\beta}
$$

where

$$
\begin{aligned}
& H_{11}=\left(\frac{v}{\left(a_{1}^{1}\right)^{2}\left(a_{2}^{2}\right)^{2}}\right)^{2 / 3}\left(a_{1}^{1}\right)^{2}, \\
& H_{22}=\left(\frac{v}{\left(a_{1}^{1}\right)^{2}\left(a_{2}^{2}\right)^{2}}\right)^{2 / 3}\left(\left(a_{2}{ }^{1}\right)^{2}+\left(a_{2}{ }^{2}\right)^{2}\right), \\
& H_{12}=\left(\frac{v}{\left(a_{1}^{1}\right)^{2}\left(a_{2}^{2}\right)^{2}}\right)^{2 / 3} a_{1}^{1} a_{2}{ }^{1}, \\
& H_{33}=\left(\frac{v}{\left(a_{1}^{1}\right)^{2}\left(a_{2}^{2}\right)^{2}}\right)^{2 / 3}\left(a_{1}^{1}\right)^{2}\left(a_{2}{ }^{2}\right)^{2} .
\end{aligned}
$$

The inverse is also useful;

$$
a_{1}{ }^{1}=H_{33} \sqrt{\frac{H_{11}}{H}}, a_{2}{ }^{1}=\frac{H_{33} H_{12}}{\sqrt{H H_{11}}}, a_{2}{ }^{2}=\sqrt{\frac{H_{33}}{H_{11}}}, v=\sqrt{H},
$$

where $H \equiv \operatorname{det}\left(H_{\alpha \beta}\right)=\left(H_{11} H_{22}-\left(H_{12}\right)^{2}\right) H_{33}$. Now, we can think of the set of metric components $\left(H_{11}, H_{22}, H_{12}, H_{33}\right)$, which are functions of $t$, as an alternative set of the dynamical variables. In such a case, the geometrical meaning of them should be understood with respect to Eq.(34).

We can also find the diffeomorphism $\psi_{\mathbf{g}}$ on ${ }^{(4)} \tilde{M}$ defined by Eq.(12), which is given by

$$
\psi_{\mathbf{g} *}:\left(\begin{array}{c}
\sigma^{1} \\
\sigma^{2} \\
\sigma^{3}
\end{array}\right) \rightarrow\left(\begin{array}{ccc}
g_{1}{ }^{1} & g_{2}{ }^{1} & 0 \\
g_{1}{ }^{2} & g_{2}{ }^{2} & 0 \\
0 & 0 & \bar{g}_{3}^{3}
\end{array}\right)\left(\begin{array}{c}
\sigma^{1} \\
\sigma^{2} \\
\sigma^{3}
\end{array}\right)
$$

or 


$$
\psi_{\mathbf{g}}:\left(\begin{array}{c}
x \\
y \\
z
\end{array}\right) \rightarrow\left(\begin{array}{c}
g_{1}{ }^{1} x+g_{2}{ }^{1} y \\
g_{1}{ }^{2} x+g_{2}{ }^{2} y \\
(1 / 2)\left(g_{1}{ }^{1} g_{1}{ }^{2} x^{2}+g_{2}{ }^{1} g_{2}{ }^{2} y^{2}\right)+g_{1}{ }^{2} g_{2}{ }^{1} x y+\bar{g}_{3}{ }^{3} z
\end{array}\right)
$$

where $\bar{g}_{3}{ }^{3} \equiv g_{1}{ }^{1} g_{2}{ }^{2}-g_{1}{ }^{2} g_{2}{ }^{1}$. In fact, we can easily calculate

$$
\psi_{\mathbf{g}} \circ A_{0} \circ \psi_{\mathbf{g}}^{-1}=\left\{\left(\begin{array}{c}
g_{1}{ }^{1} \\
g_{1}{ }^{2} \\
\frac{1}{2} g_{1}{ }^{1} g_{1}{ }^{2}
\end{array}\right),\left(\begin{array}{c}
g_{2}{ }^{1} \\
g_{2}{ }^{2} \\
\frac{1}{2} g_{2}{ }^{1} g_{2}{ }^{2}
\end{array}\right),\left(\begin{array}{c}
0 \\
0 \\
\bar{g}^{3}{ }_{3}
\end{array}\right)\right\},
$$

which coincides with $\Gamma_{\mathbf{g}}$ given in Eq.(18) $)_{\mathrm{II}}$, up to unessential conjugation, again. (Note that Eq.(36) corresponds to a case of $f^{3}{ }_{1}=f^{3}{ }_{2}=0$ for the general HPDs (22). The spacetime metric with $f^{3}{ }_{1}$ and $f^{3}{ }_{2}$ as constant parameters was possible as a solution of Einstein's equation, but the space spanned by $f^{3}{ }_{1}$ and $f_{2}^{3}$ in the space of solutions corresponds to the gauge orbits generated by the momentum constraints, so that they cannot be true dynamical degrees of freedom.)

If we make the dynamical metric following Eq.(13) (with the universal cover metric (6)), and substitute into Eq.(35), we obtain the time development in terms of $\mathbf{h}$ of the dynamical variables, previously obtained in II (see Eqs.(31) II and (32) II). This guarantees the rightness of the dynamical metric (33).

Finally, our phase space $P$ is spanned by $\left(a_{1}{ }^{1}, a_{2}{ }^{1}, a_{2}{ }^{2}, v, p^{1}{ }_{1}, p^{2}{ }_{1}, p^{2}{ }_{2}, p_{v}\right)$, where $p^{i}{ }_{j}$ and $p_{v}$ are the conjugate momenta of $a_{i}{ }^{j}$ and $v$, respectively. The Hamiltonian on $P$ is naturally obtained from the dynamical metric (33) and the usual Einstein-Hilbert action as

$$
\begin{aligned}
\mathcal{H}= & \frac{1}{2 v}\left\{\left(a_{1}{ }^{1} a_{2}{ }^{2} v\right)^{4 / 3}+\left(a_{1}{ }^{1}\right)^{2}\left(p^{1}{ }_{1}\right)^{2}+\left(\left(a_{2}{ }^{1}\right)^{2}+\left(a_{2}{ }^{2}\right)^{2}\right)\left(p^{2}{ }_{1}\right)^{2}+\left(a_{2}{ }^{2}\right)^{2}\left(p^{2}{ }_{2}\right)^{2}\right. \\
& \left.+2 a_{1}{ }^{1} a_{2}{ }^{1} p^{1}{ }_{1} p^{2}{ }_{1}+a_{1}{ }^{1} a_{2}{ }^{2} p^{1}{ }_{1} p^{2}{ }_{2}+a_{2}{ }^{1} a_{2}{ }^{2} p^{2}{ }_{1} p^{2}{ }_{2}\right\}-\frac{3}{8} v\left(p_{v}\right)^{2} .
\end{aligned}
$$

The Hamiltonian constraint, given by $\mathcal{H} \approx 0$, reduces the dynamical degrees of freedom from $\operatorname{dim} P=8$ to six, which agrees with the count given in II, i.e., two for $\mathbf{u}$, and four for $\mathbf{g}=\left\{g_{1}{ }^{1}, g_{1}{ }^{2}, g_{2}{ }^{1}, g_{2}{ }^{2}\right\}$.

\section{B. The f1/1 $(n)$ model}

As in Bianchi II, the HPDs $\eta$ of Bianchi $\mathrm{VI}_{0}$ are obtained from the invariance under Eq.(5) of the Maurer-Cartan relation

$$
\mathrm{d} \sigma^{1}=\sigma^{2} \wedge \sigma^{3}, \quad \mathrm{~d} \sigma^{2}=-\sigma^{3} \wedge \sigma^{1}, \quad \mathrm{~d} \sigma^{3}=0,
$$

with respect to the invariant 1 -forms $\sigma^{i}$ of Bianchi $\mathrm{VI}_{0}$, given by 


$$
\sigma^{1} \equiv \frac{1}{\sqrt{2}}\left(e^{z} \mathrm{~d} x+e^{-z} \mathrm{~d} y\right), \sigma^{2} \equiv \frac{1}{\sqrt{2}}\left(-e^{z} \mathrm{~d} x+e^{-z} \mathrm{~d} y\right), \sigma^{3} \equiv \mathrm{d} z
$$

We find

$$
\eta_{*}:\left(\begin{array}{c}
\sigma^{1} \\
\sigma^{2} \\
\sigma^{3}
\end{array}\right) \rightarrow\left(\begin{array}{ccc}
f_{1}^{1} & f^{1}{ }_{2} & f^{1}{ }_{3} \\
\pm f_{2}^{1} & \pm f^{1}{ }_{1} & f^{2}{ }_{3} \\
0 & 0 & \pm 1
\end{array}\right)\left(\begin{array}{c}
\sigma^{1} \\
\sigma^{2} \\
\sigma^{3}
\end{array}\right)
$$

We need only the identity component of the HPDs, which correspond to the plus signs in Eq.(42). Integrating $\eta_{*}$ for the plus signs, we have

$$
\eta:\left(\begin{array}{c}
x \\
y \\
z
\end{array}\right) \rightarrow\left(\begin{array}{c}
\left(f_{1}^{1}-f_{2}^{1}\right) x-\frac{1}{\sqrt{2}}\left(f_{3}^{1}-f_{3}^{2}\right) e^{-z} \\
\left(f_{1}^{1}+f_{2}^{1}\right) y+\frac{1}{\sqrt{2}}\left(f_{3}^{1}+f_{3}^{2}\right) e^{z} \\
z
\end{array}\right)
$$

We have again set the integral constants zero.

Our standard universal cover metric is (cf. Eq.(80) $\mathrm{I}$ )

$$
\mathrm{d} l^{2}=e^{2 \lambda}\left(\sigma^{1}\right)^{2}+e^{-2 \lambda}\left(\sigma^{2}\right)^{2}+\left(\sigma^{3}\right)^{2}
$$

and our parameterization of the Teichmüller space is given by the following generators acting on the standard universal cover (cf. Eqs.(156) I and (157) I);

$$
A_{\boldsymbol{\tau}}=\left\{\left(\begin{array}{c}
\alpha u_{1} \\
\alpha u_{2} \\
0
\end{array}\right),\left(\begin{array}{c}
\alpha v_{1} \\
\alpha v_{2} \\
0
\end{array}\right),\left(\begin{array}{c}
0 \\
0 \\
c_{3}
\end{array}\right)\right\}, \quad \text { for } n>2,
$$

and

$$
A_{\boldsymbol{\tau}}=\left\{\left(\begin{array}{c}
\alpha u_{1} \\
\alpha u_{2} \\
0
\end{array}\right),\left(\begin{array}{c}
\alpha v_{1} \\
\alpha v_{2} \\
0
\end{array}\right), h \circ\left(\begin{array}{c}
0 \\
0 \\
c_{3}
\end{array}\right)\right\}, \quad \text { for } n<-2,
$$

where $e^{c_{3}} \equiv\left|n+\sqrt{n^{2}-4}\right| / 2$,

$$
\left(\begin{array}{l}
u_{1} \\
v_{1}
\end{array}\right) \equiv \frac{1}{\sqrt{|n|}}\left(\begin{array}{l}
\sqrt{\frac{\left|n+\sqrt{n^{2}-4}\right|}{2}} \\
\sqrt{\frac{\left|n-\sqrt{n^{2}-4}\right|}{2}}
\end{array}\right),\left(\begin{array}{l}
u_{2} \\
v_{2}
\end{array}\right) \equiv \frac{1}{\sqrt{|n|}}\left(\begin{array}{l}
\frac{\sqrt{\frac{\left|n-\sqrt{n^{2}-4}\right|}{2}}}{\sqrt{\frac{\left|n+\sqrt{n^{2}-4}\right|}{2}}}
\end{array}\right)
$$

and $h$ is defined by $h:(x, y, z) \rightarrow(-x,-y, z)$. So, we have

$$
\boldsymbol{r}=\{\lambda\}, \quad \boldsymbol{\tau}=\{\alpha\}
$$

Adding the volume $v$, we have three dynamical variables $(\boldsymbol{r}, \boldsymbol{\tau}, v)$. 
It is quite easy to find the (HP)TDs, if noting the fact that the compact manifold $\mathrm{f} 1 / 1(n)$ is a torus bundle over the circle. Since the Teichmüller parameter $\alpha$ is the size of the torus fiber, laid in $x-y$ plane, relative to the circle, laid in z-axis, the HPTDs are supposed to be

$$
\phi_{\boldsymbol{\tau}}:(x, y, z) \rightarrow(\alpha x, \alpha y, z) .
$$

This is apparently an element of the HPDs (43), i.e., corresponding to the case of $f^{1}{ }_{1}=$ $\alpha, f^{1}{ }_{2}=f^{1}{ }_{3}=f^{2}{ }_{3}=0$. We can even make sure the relation (8) if letting $\boldsymbol{\tau}_{0}=\{1\}$. The diffeomorphisms (49) are therefore the HPTDs.

As in the b/1 case, normalizing the induced metric of Eq.(44) to give $v$ as its volume element, we obtain the dynamical metric

$$
\mathrm{d} s^{2}=-\mathrm{d} t^{2}+H_{11}\left(\sigma^{1}\right)^{2}+H_{22}\left(\sigma^{2}\right)^{2}+H_{33}\left(\sigma^{3}\right)^{2},
$$

where

$$
\begin{aligned}
& H_{11}=\left(v^{2} \alpha^{-4}\right)^{1 / 3} e^{2 \lambda} \alpha^{2}, \\
& H_{22}=\left(v^{2} \alpha^{-4}\right)^{1 / 3} e^{-2 \lambda} \alpha^{2}, \\
& H_{33}=\left(v^{2} \alpha^{-4}\right)^{1 / 3} .
\end{aligned}
$$

The inverse is also useful;

$$
\alpha=\frac{\left(H_{11} H_{22}\right)^{1 / 4}}{\sqrt{H_{33}}}, \lambda=\frac{1}{4} \ln \frac{H_{11}}{H_{22}}, v=\sqrt{H_{11} H_{22} H_{33}} .
$$

We can easily check that the $\psi_{\mathbf{g}}$ defined by Eq.(12) with $\mathbf{g}=\left\{\alpha_{0}\right\}$ is given by

$$
\psi_{\mathbf{g}}:(x, y, z) \rightarrow\left(\alpha_{0} x, \alpha_{0} y, z\right) .
$$

In the same manner as the b/1 case, we obtain the time development of the dynamical variables,

$$
\alpha=\frac{\left(h_{11} h_{22}\right)^{1 / 4}}{\sqrt{h_{33}}} \alpha_{0}, \quad \lambda=\frac{1}{4} \ln \frac{h_{11}}{h_{22}}, \quad v=\sqrt{h_{11} h_{22} h_{33}}\left(\alpha_{0}\right)^{2},
$$

which coincides with Eq.(46) II. Again, this guarantees the rightness of the dynamical metric (33). (There are factor or power errors in the last paragraph of Sec.IV B of II, for the values of $\lambda$ and $v$.)

Our phase space $P$ is spanned by $\left(\lambda, \alpha, v, p_{\lambda}, p_{\alpha}, p_{v}\right)$, where $p_{\lambda}, p_{\alpha}$ and $p_{v}$ are the conjugate momenta of $\lambda, \alpha$ and $v$, respectively. The Hamiltonian on $P$ is naturally obtained from the dynamical metric (50) as

$$
\mathcal{H}=\frac{1}{2 v}\left\{4 \cosh ^{2} 2 \lambda(\alpha v)^{4 / 3}+\frac{1}{4} p_{\lambda}^{2}+\frac{3}{4} \alpha^{2} p_{\alpha}^{2}\right\}-\frac{3}{8} v\left(p_{v}\right)^{2} .
$$

The Hamiltonian constraint $\mathcal{H} \approx 0$ reduces the dynamical degrees of freedom from $\operatorname{dim} P=6$ to four, which agrees with the count given in II, i.e., three for $\mathbf{u}$, and one for $\mathbf{g}=\left\{\alpha_{0}\right\}$. 


\section{The a1/1 model}

The pullback of HPDs for Bianchi $\mathrm{VII}_{0}$ are found to be

$$
\eta_{*}:\left(\begin{array}{c}
\sigma^{1} \\
\sigma^{2} \\
\sigma^{3}
\end{array}\right) \rightarrow\left(\begin{array}{ccc}
f^{1}{ }_{1} & f^{1}{ }_{2} & f^{1}{ }_{3} \\
\mp f^{1}{ }_{2} & \pm f^{1}{ }_{1} & f^{2}{ }_{3} \\
0 & 0 & \pm 1
\end{array}\right)\left(\begin{array}{c}
\sigma^{1} \\
\sigma^{2} \\
\sigma^{3}
\end{array}\right)
$$

for the invariant 1-forms defined by

$$
\sigma^{1}=\cos z \mathrm{~d} x+\sin z \mathrm{~d} y, \sigma^{2}=-\sin z \mathrm{~d} x+\cos z \mathrm{~d} y, \sigma^{3}=\mathrm{d} z
$$

We need only the identity component of the HPDs, which correspond to the upper set of signs in Eq.(56). Integrating $\eta_{*}$ for the identity component, and setting the integral constants zero, we obtain the following HPDs;

$$
\eta:\left(\begin{array}{c}
x \\
y \\
z
\end{array}\right) \rightarrow\left(\begin{array}{c}
f^{1}{ }_{1} x+f^{1}{ }_{2} y+f^{1}{ }_{3} \sin z+f^{2}{ }_{3} \cos z \\
-f^{1}{ }_{2} x+f^{1}{ }_{1} y-f^{1}{ }_{3} \cos z+f^{2}{ }_{3} \sin z \\
z
\end{array}\right)
$$

Our standard universal cover metric is (cf. Eq.(72) I )

$$
\mathrm{d} l^{2}=e^{2 \lambda}\left(\sigma^{1}\right)^{2}+e^{-2 \lambda}\left(\sigma^{2}\right)^{2}+\left(\sigma^{3}\right)^{2}
$$

and our parameterization of the Teichmüller space is given by the following generators acting on the standard universal cover (cf. Eq.(100)I);

$$
A_{\boldsymbol{\tau}}=\left\{\left(\begin{array}{c}
a_{1}{ }^{1} \\
0 \\
2 l \pi
\end{array}\right),\left(\begin{array}{c}
a_{2}{ }^{1} \\
a_{2}{ }^{2} \\
2 m \pi
\end{array}\right),\left(\begin{array}{c}
a_{3}{ }^{1} \\
a_{3}{ }^{2} \\
2 n \pi
\end{array}\right)\right\}
$$

where $l, m$, and $n$ are integers. (The choice of Eq.(101) I will give the same dynamical metric as that of Eq.(100)I, since they differ by just a discrete element. So, we focus on the case of Eq.(60).)

Note that we have seven variables $(\boldsymbol{r}, \boldsymbol{\tau}, v)$, where

$$
\boldsymbol{r}=\{\lambda\}, \quad \boldsymbol{\tau}=\left\{a_{1}{ }^{1}, a_{2}{ }^{1}, a_{2}{ }^{2}, a_{3}{ }^{1}, a_{3}{ }^{2}\right\}
$$

and $v$ is the volume. We know, however, that all the Teichmüller parameters $\boldsymbol{\tau}$ develop in time in the same manner (see Eq.(54) II);

$$
a_{i}{ }^{j}=\frac{\left(h_{11} h_{22}\right)^{1 / 4}}{\sqrt{h_{33}}} g_{i}{ }^{j},(i, j)=(1,1),(2,1),(2,2),(3,1), \text { and }(3,2),
$$


where $\mathbf{g} \equiv\left\{g_{i}{ }^{j}\right\}$ are the constant parameters in the $\Gamma_{\mathbf{g}}$ (cf. Eq.(50) II $_{\text {). Th may suggest }}$ that dynamically, and at least classically, the five Teichmüller parameters degenerate and we should think of only one of them as a true dynamical variable, though geometrically all of them reflect true degrees of freedom of smooth deformations of the compact manifold a1/1. We in this subsection take this standpoint, since only by doing so we can succeed to have the dynamical spacetime metric. (See below.)

Now, our dynamical variables are $\left(\boldsymbol{r}, \boldsymbol{\tau}^{\prime}, v\right)$, where $\boldsymbol{\tau}^{\prime}=\{a\}$ is one of the elements in $\boldsymbol{\tau}$. We also define $\mathbf{g}^{\prime} \equiv\{g\}$, which is one of $\mathbf{g}$, corresponding to $a$. The HPTDs, the $\psi_{\mathbf{g}^{\prime}}$, and the dynamical spacetime metric are most similar to those of the f1/1(n) model. The HPTDs are given by

$$
\phi_{\boldsymbol{\tau}^{\prime}}:(x, y, z) \rightarrow(a x, a y, z),
$$

which is a case of $f^{1}{ }_{2}=f^{1}{ }_{3}=f^{2}{ }_{3}=0$ of Eq.(58). The $\psi_{\mathbf{g}^{\prime}}$ is given by

$$
\psi_{\mathbf{g}^{\prime}}:(x, y, z) \rightarrow(g x, g y, z) .
$$

The dynamical spacetime metric is given by

$$
\mathrm{d} s^{2}=-\mathrm{d} t^{2}+H_{11}\left(\sigma^{1}\right)^{2}+H_{22}\left(\sigma^{2}\right)^{2}+H_{22}\left(\sigma^{2}\right)^{2},
$$

where

$$
\begin{aligned}
& H_{11}=\left(v^{2} a^{-4}\right)^{1 / 3} e^{2 \lambda} a^{2}, \\
& H_{22}=\left(v^{2} a^{-4}\right)^{1 / 3} e^{-2 \lambda} a^{2}, \\
& H_{33}=\left(v^{2} a^{-4}\right)^{1 / 3} .
\end{aligned}
$$

Then, as in the f1/1(n) case, we should have

$$
a=\frac{\left(h_{11} h_{22}\right)^{1 / 4}}{\sqrt{h_{33}}} g,
$$

which reproduces (a representative of) Eq.(62). In this sense, metric (65) is the right dynamical metric. This diagonal type of metric was, in fact, the only possibility of the dynamical metric made from the HPTDs, since if we wrote the general Bianchi $\mathrm{VII}_{0}$ spacetime metric, the off-diagonal three components would have become nondynamical, due to the three momentum constraints $[8]$.

Our phase space $P$ is spanned by $\left(\lambda, a, v, p_{\lambda}, p_{a}, p_{v}\right)$, where $p_{\lambda}, p_{a}$ and $p_{v}$ are the conjugate momenta of $\lambda, a$ and $v$, respectively. The Hamiltonian on $P$ is naturally obtained from the dynamical metric (65) as

$$
\mathcal{H}=\frac{1}{2 v}\left\{4 \sinh ^{2} 2 \lambda(a v)^{4 / 3}+\frac{1}{4} p_{\lambda}^{2}+\frac{3}{4} a^{2} p_{a}^{2}\right\}-\frac{3}{8} v\left(p_{v}\right)^{2} .
$$

The Hamiltonian constraint $\mathcal{H} \approx 0$ reduces the dynamical degrees of freedom from $\operatorname{dim} P=6$ to four, which does not, of course, agree with the count given in II, because variable $a$ is a representative of the five Teichmüller parameters $a_{i}{ }^{j}$. 


\section{The a2/1 (flat torus) model}

Both the HPDs for Bianchi I and the pullback of them are the general linear transformations;

$$
\eta: x^{\mu} \rightarrow \sum_{\nu} f^{\mu}{ }_{\nu} x^{\nu} ; \quad \eta_{*}: \sigma^{\mu} \rightarrow \sum_{\nu} f^{\mu}{ }_{\nu} \sigma^{\nu}
$$

where $\operatorname{det}\left(f^{\mu}{ }_{\nu}\right) \neq 0,\left(x^{1}, x^{2}, x^{3}\right) \equiv(x, y, z)$, and $\left(\sigma^{1}, \sigma^{2}, \sigma^{3}\right) \equiv(\mathrm{d} x, \mathrm{~d} y, \mathrm{~d} z)$.

Our standard universal cover is simply (cf. Eq.(66) I)

$$
\mathrm{d} l^{2}=\left(\sigma^{1}\right)^{2}+\left(\sigma^{2}\right)^{2}+\left(\sigma^{3}\right)^{2}
$$

and our parameterization of the Teichmüller space is given by the following generators acting on the standard universal cover;

$$
A_{\boldsymbol{\tau}}=\left\{\left(\begin{array}{c}
a_{1}^{1} \\
0 \\
0
\end{array}\right),\left(\begin{array}{c}
a_{2}{ }^{1} \\
a_{2}^{2} \\
0
\end{array}\right),\left(\begin{array}{c}
a_{3}^{1} \\
a_{3}^{2} \\
a_{3}{ }^{3}
\end{array}\right)\right\} .
$$

That is, we have no curvature parameter and six Teichmüller parameters;

$$
\boldsymbol{r}=\emptyset, \quad \boldsymbol{\tau}=\left\{a_{1}{ }^{1}, a_{2}{ }^{1}, a_{2}{ }^{2}, a_{3}{ }^{1}, a_{3}{ }^{2}, a_{3}{ }^{3}\right\}
$$

We choose $\boldsymbol{\tau}_{0}=\{1,0,1,0,0,1\}$, for later convenience. In the parameterization (71), the dynamical variables are only the Teichmüller parameters $\boldsymbol{\tau}$; the freedom of volume variations are contained in them. This is due to the fact that a conformal transformation on the standard universal cover with constant conformal factor is just the pullback of a diffeomorphism on the cover. This occurs only in the case of Bianchi I. If one wants to factor out the volume $v$ from the dynamical variables, one can do so by normalizing $\boldsymbol{\tau}$ to give the unit volume for the quotient, but we don't as in II.

$$
\begin{aligned}
& \text { Let } \mathbf{a}=\left(\begin{array}{l}
a^{1} \\
a^{2} \\
a^{3}
\end{array}\right), \mathbf{x}=\left(\begin{array}{l}
x \\
y \\
z
\end{array}\right) \in \text { BI. Then, } \\
& \qquad \begin{aligned}
\eta \circ \mathbf{a} \circ \eta^{-1} \circ \mathbf{x} & =\eta \circ \mathbf{a} \circ\left(f^{-1} \mathbf{x}\right) \\
& =\eta \circ\left(\mathbf{a}+f^{-1} \mathbf{x}\right) \\
& =f \mathbf{a}+\mathbf{x},
\end{aligned}
\end{aligned}
$$

where $f \equiv\left(f^{\mu}{ }_{\nu}\right)$ is the matrix composed of $f^{\mu}{ }_{\nu}$, and the usual multiplication rule for matrix is understood whenever "o" does not appear. Thus, the induced element of a by $\eta$ is the linear transformation of $\mathbf{a}$; 


$$
\eta \circ \mathbf{a} \circ \eta^{-1}=f \mathbf{a} .
$$

The TDs must satisfy Eq.(8), which is now equivalent to

$$
f M_{0}=M_{\boldsymbol{\tau}}
$$

where $M_{\boldsymbol{\tau}}$ is the matrix composed of the generators of $A_{\boldsymbol{\tau}}$;

$$
M_{\boldsymbol{\tau}} \equiv\left(\begin{array}{ccc}
a_{1}{ }^{1} & a_{2}{ }^{1} & a_{3}{ }^{1} \\
0 & a_{2}{ }^{2} & a_{3}{ }^{2} \\
0 & 0 & a_{3}{ }^{3}
\end{array}\right) \text {. }
$$

Since $M_{0}$ is the identity matrix, this implies

$$
f=M_{\boldsymbol{\tau}} .
$$

We have found the TDs in the HPDs, so this is the HPTDs. The pullback of the standard metric (70) by these HPTDs immediately gives (the spatial part of) the dynamical metric; we have

$$
\mathrm{d} s^{2}=-\mathrm{d} t^{2}+H_{\alpha \beta} \sigma^{\alpha} \sigma^{\beta}
$$

where

$$
\left(H_{\alpha \beta}\right)=\left(\sum_{\gamma} M_{\boldsymbol{\tau}_{\gamma \alpha}} M_{\boldsymbol{\tau}_{\gamma \beta}}\right)=\left(\begin{array}{ccc}
\left(a_{1}{ }^{1}\right)^{2} & a_{1}{ }^{1} a_{2}{ }^{1} & a_{1}{ }^{1} a_{3}{ }^{1} \\
& \left(a_{2}{ }^{1}\right)^{2}+\left(a_{2}{ }^{2}\right)^{2} & a_{2}{ }^{1} a_{3}{ }^{1}+a_{2}{ }^{2} a_{3}{ }^{2} \\
(\text { sym. }) & & \left(a_{3}{ }^{1}\right)^{2}+\left(a_{3}{ }^{2}\right)^{2}+\left(a_{3}{ }^{3}\right)^{2}
\end{array}\right) .
$$

Now, the metric components $\left(H_{11}, H_{12}, H_{13}, H_{22}, H_{23}, H_{33}\right)$ are an alternative set of the dynamical variables $\tau$. We can use the general Bianchi I spacetime metric (78) as the universal cover metric of the flat torus (a2/1) universe. In such a case, the geometrical interpretation of the components should be understood with respect to Eq.(79).

The $\psi_{\mathbf{g}}$ defined by Eq. (12) is found to be

$$
\psi_{\mathbf{g} *}: \sigma^{\mu} \rightarrow \sum_{\nu} g_{\nu}{ }^{\mu} \sigma^{\nu}
$$

or

$$
\psi_{\mathbf{g}}: x^{\mu} \rightarrow \sum_{\nu} g_{\nu}{ }^{\mu} x^{\nu}
$$

In fact, we can easily check that Eq.(12) holds for the $\Gamma_{\mathrm{g}}$ given by (cf. Eq.(58)

$$
\Gamma_{\mathbf{g}}=\left\{\left(\begin{array}{c}
g_{1}{ }^{1} \\
g_{1}{ }^{2} \\
g_{1}{ }^{3}
\end{array}\right),\left(\begin{array}{c}
g_{2}{ }^{1} \\
g_{2}{ }^{2} \\
g_{2}{ }^{3}
\end{array}\right),\left(\begin{array}{l}
g_{3}{ }^{1} \\
g_{3}{ }^{2} \\
g_{3}{ }^{3}
\end{array}\right)\right\}
$$


If we make the dynamical metric following Eq.(13) (with the universal cover metric (6)), the spatial components $H_{\alpha \beta}$ are found to be $H_{\alpha \beta}=\sum_{\mu, \nu} h_{\mu \nu} g_{\alpha}{ }^{\mu} g_{\beta}{ }^{\nu}$. Here, $h_{\mu \nu}=$ $\operatorname{diag}\left(t^{2 p_{1}}, t^{2 p_{2}}, t^{2 p_{3}}\right)$, and $p_{\alpha}$ 's are constant parameters satisfying $p_{1}+p_{2}+p_{3}=1=$ $\left(p_{1}\right)^{2}+\left(p_{2}\right)^{2}+\left(p_{3}\right)^{2}$, for vacuum. Comparison with Eq.([9) will give the same time development of the Teichmüller parameters as Eq.(65) II, which fact justifies the use of our dynamical metric.

Finally, our phase space $P$ is spanned by the six $a_{i}{ }^{j}$ 's and the corresponding conjugate momenta $p_{j}^{i}$ 's. Though the present parameterization may not be convenient for practical purpose, we, for completeness, present the Hamiltonian on $P$, which is naturally obtained from the dynamical metric (78) with Eq.(79);

$$
\begin{aligned}
\mathcal{H}=\frac{1}{8 a_{1}{ }^{1} a_{2}{ }^{2} a_{3}{ }^{3}} & \left\{\left(a_{1}{ }^{1} p^{1}{ }_{1}+a_{2}{ }^{1} p^{2}{ }_{1}-a_{2}{ }^{2} p^{2}{ }_{2}+a_{3}{ }^{1} p^{3}{ }_{1}-a_{3}{ }^{2} p^{3}{ }_{2}-a_{3}{ }^{3} p^{3}{ }_{3}\right)^{2}\right. \\
+ & 4\left(a_{2}{ }^{2} p^{2}{ }_{1}+a_{3}{ }^{2} p^{3}{ }_{1}\right)^{2}+4\left(a_{3}{ }^{3}\right)^{2}\left(\left(p^{3}{ }_{1}\right)^{2}+\left(p^{3}{ }_{2}\right)^{2}\right) \\
& \left.-4 a_{3}{ }^{3} p^{3}{ }_{3}\left(a_{2}{ }^{2} p^{2}{ }_{2}+a_{3}{ }^{2} p^{3}{ }_{2}\right)\right\} .
\end{aligned}
$$

The Hamiltonian constraint $\mathcal{H} \approx 0$ reduces the dynamical degrees of freedom from $\operatorname{dim} P=12$ to ten, which agrees with the count given in II, i.e., one for $\mathbf{u}$ (the inde-

pendent Kasner parameter), and nine for $\mathbf{g}=\left\{g_{\mu}{ }^{\nu}\right\}$ (the constant parameters needed for the compactification).

\section{CONCLUDING REMARKS}

We have defined a class of diffeomorphisms, called Teichmüller diffeomorphisms(TDs), which induce the Teichmüller deformations of the quotient, in conjunction with a fixed covering group. A subclass of the TDs, called the HPTDs, has also been defined. They match the dynamical evolution of the Teichmüller parameters. We have obtained the Hamiltonians

for the space $P=T_{*} F$, by restricting the spacetime metric to the dynamical metric, i.e., to the metric induced by the HPTDs.

The Hamiltonian structure thus found is the same as the one which would have obtained from the general Bianchi metric which is free from the momentum constraints. However [8], the dynamical degrees of freedom do not have clear meaning in the context of the usual open model. In our compact model, in contrast to this, all the dynamical variables, and thus all the dynamical degrees of freedom, have explicit geometrical meaning, since our approach is "constructive". One can, nevertheless, also regard our results as giving geometrical interpretation of the known Hamiltonian for a Bianchi metric.

Our approach will be applicable to larger classes of spacetimes, e.g., spacetimes of which space is non compact locally homogeneous but has nontrivial fundamental group, and spacetimes of which space possesses less than three local Killing vectors. One in the former case 
has less Teichmüller parameters than the compact case. Whether a consistent Hamiltonian is obtained or not will, however, depend upon each model.

We end this final section with some remarks on the compact models on Bianchi VIII, i.e., compact models such that (the identity component of) the extendible isometry group is given by the Bianchi VIII group, i.e., Esom ${ }_{0} \tilde{M}_{t}=$ BVIII. We simply call these models the "d/*" models, where, as usual, d stands for the type of the universal cover and $*$ is a characterization of the compact quotient. (See I, for explicit description of "*".) For definiteness, we show the standard universal cover metric, up to constant conformal factor, of the $\mathrm{d} / *$ models; it is given by

$$
\mathrm{d} l^{2}=e^{2\left(\lambda_{+} / \sqrt{3}+\lambda_{-}\right)}\left(\sigma^{1}\right)^{2}+e^{2\left(\lambda_{-} / \sqrt{3}-\lambda_{-}\right)}\left(\sigma^{2}\right)^{2}+e^{-(4 / \sqrt{3}) \lambda_{+}}\left(\sigma^{3}\right)^{2},
$$

where $\lambda_{+}$and $\lambda_{-}$are constants, and the invariant 1 -forms $\sigma^{\mu}$ are

$$
\sigma^{1}=\frac{1}{y}(\sin z \mathrm{~d} x-\cos z \mathrm{~d} y), \quad \sigma^{2}=\frac{1}{y}(\cos z \mathrm{~d} x+\sin z \mathrm{~d} y), \quad \sigma^{3}=\frac{1}{y}(\mathrm{~d} x+y \mathrm{~d} z) .
$$

(The present choice of the invariant 1-forms differs from the one listed in I. This is, of course, a matter of preference. But it was an erroneous claim of I that [14] to admit a compact quotient, the universal cover should admit four dimensional isometry group and therefore the $\lambda_{-}$should vanish. Actually, there exist many cases such that we can embed the fundamental group $\pi_{1}(\mathrm{~d} / *)$ into BVIII itself with the discrete but generic isometries $j: z \rightarrow z+2 n \pi$, where $n$ is an integer. The coefficients of the invariant 1-forms (i.e., the curvature parameters) in Tables I and II of Paper I should, accordingly, read as Eq.(84), and the "Degrees of freedom of the universal cover U" in Table III of the same paper should read "2".)

We know that the vacuum solution of the conventional Bianchi VIII model is diagonalizable (e.g. Ref. [2]), which means that the universal cover metric $\tilde{\mathrm{g}}_{a b}[\mathbf{u}]$ of the spacetime approach is of the form of Eq.(何). So, its spatial part $\tilde{h}_{a b}[\mathbf{u}]$ is also diagonal. On the other hand, the standard metric $\tilde{h}_{a b}^{\text {std }}[\boldsymbol{r}]=e^{2 \alpha}\left(\mathrm{d} l^{2}\right)_{a b}$, where $\mathrm{d} l^{2}$ is given by Eq.(84) and $e^{2 \alpha}$ is a conformal factor, is of the diagonal form of which three components can be freely specified. So, the spatial metric $\tilde{h}_{a b}[\mathbf{u}]$ can be directly identified with the standard metric, i.e., $\tilde{h}_{a b}^{\mathrm{std}}[\boldsymbol{r}]=\tilde{h}_{a b}[\mathbf{u}]$. Moreover, since it holds $\operatorname{Esom} \tilde{M}_{t}=\operatorname{Isom} \tilde{M}_{t}$, we do not need take conjugations of $\Gamma_{\mathbf{g}}$ to have it coincide with $A_{\boldsymbol{\tau}}$. Therefore, we have $\Gamma_{\mathbf{g}}=A_{\boldsymbol{\tau}}$, implying

$$
\boldsymbol{\tau}(t)=\mathbf{g}=\text { constants. }
$$

Namely, the Teichmüller parameters of the $d / *$ models do not develop in time. In this sense, the Teichmüller parameters of the $\mathrm{d} / *$ models may be regarded as nondynamical, though we defined any Teichmüller parameter as a dynamical variable. If on this standpoint, the dynamical variables are only the curvature parameters $\left(\lambda_{+}, \lambda_{-}, \alpha\right)$, or equivalently the three 
diagonal components of the Bianchi VIII metric, so that the reduced Hamiltonian with its geometrical interpretation is trivial. In fact, since $\chi=0$ holds, the HPDs for Bianchi VIII have no freedom to store the TDs.

Note after the completion of this work: Recently, Kodama [15] has studied the canonical structure for SCH spacetimes by a different approach, the diffeomorphism-invariant phase space approach, giving consistent Hamiltonians with ours.

\section{ACKNOWLEDGMENTS}

M.T. acknowledges financial support from the Japan Society for the Promotion of Science and the Ministry of Education, Science and Culture.

[1] L. D. Landau, E. M. Lifshitz, Classical theory of Fields (MIT, Reading, 1971).

[2] M. P. Ryan, L. C. Shepley, Homogeneous Relativistic Cosmologies. Princeton Series in Physics (Princeton University Press, Princeton, 1975).

[3] R. M. Wald, General Relativity (University of Chicago, Chicago, 1984).

[4] J. J. Halliwell, in Quantum cosmology and baby universes, edited by S. Coleman, J. B. Hartle, T. Piran, and S. Weinberg, (World Scientific, Singapore, 1991), pp.159-243, and references therein.

[5] P. D. D'Eath, Supersymmetric quantum cosmology, (Cambridge University Press, Cambridge, 1996).

[6] See, e.g., S. Weinberg, Gravitation and cosmology. (Wiley, New York, 1972).

[7] V. A. Belinski, I. M. Khalatnikov and E. M. Lifshitz, Adv. in Phys. 19, 525 (1970).

[8] A. Ashtekar and J. Samuel, Class. Quantum Gravit. 8, 2191 (1991).

[9] T. Koike, M. Tanimoto and A. Hosoya, J. Math. Phys. 35, 4855 (1994).

[10] M. Tanimoto, T. Koike and A. Hosoya, J. Math. Phys. 38, 350 (1997).

[11] K. Ohshika, Topology and its Application 27, 75 (1987).

[12] R. Kulkarni, K. B. Lee, F. Raymond, Geometry and Topology, Lecture Notes Math. 1167, $180(1985)$. 
[13] A. Hosoya and K. Nakao, Class. Quantum Gravit. 7, 163 (1990). For recent status of (2+1)gravity and more references, see e.g. S. Carlip, Lectures on (2+1)-Dimensional Gravity (grqc/9503024, 1995).

[14] We thank A. Higuchi and H. Kodama for pointing out this point.

[15] H. Kodama, Canonical structure of locally homogeneous systems on compact closed 3manifolds of types $E^{3}$, Nil and Sol, to appear. 


\section{FIGURE CAPTIONS}

FIGURE 1: The unit cube (a fundamental region of $\pi_{0}$ ) and its image (a fundamental region of $\pi_{\tau}$ ) by an HPTD.

FIGURE 2: The parallelogram shows the projection of a fundamental region of $\pi_{\tau}$ on the $x-y$ plane. 


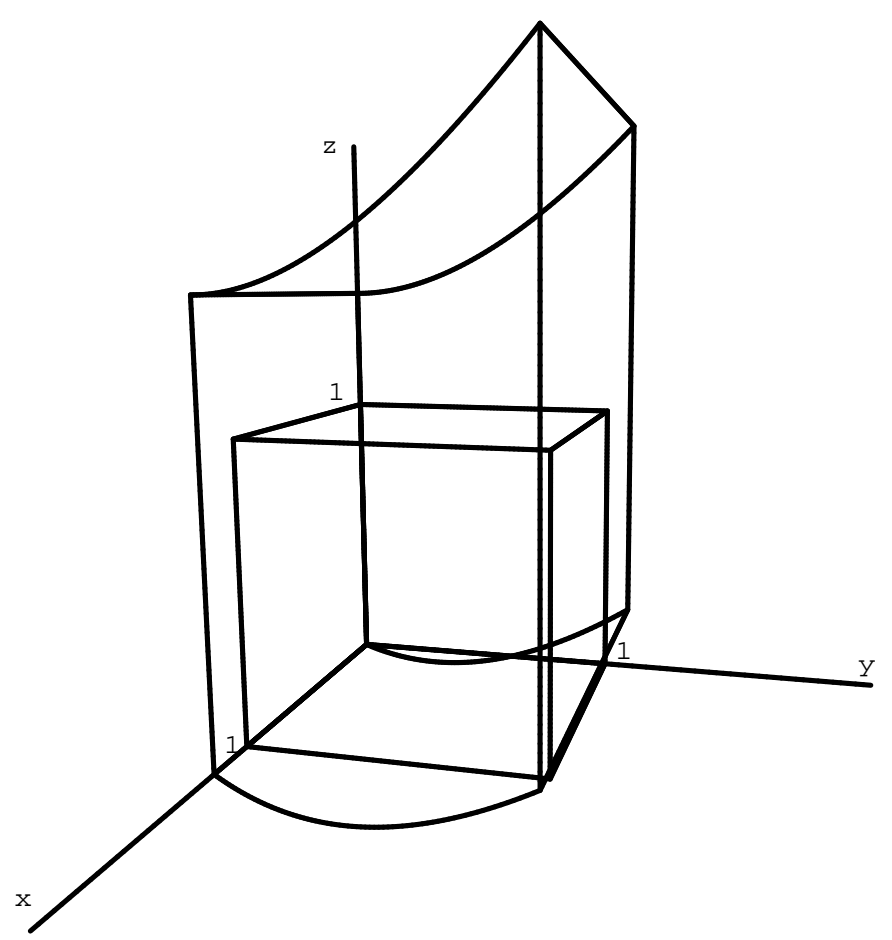

FIGURE 1 


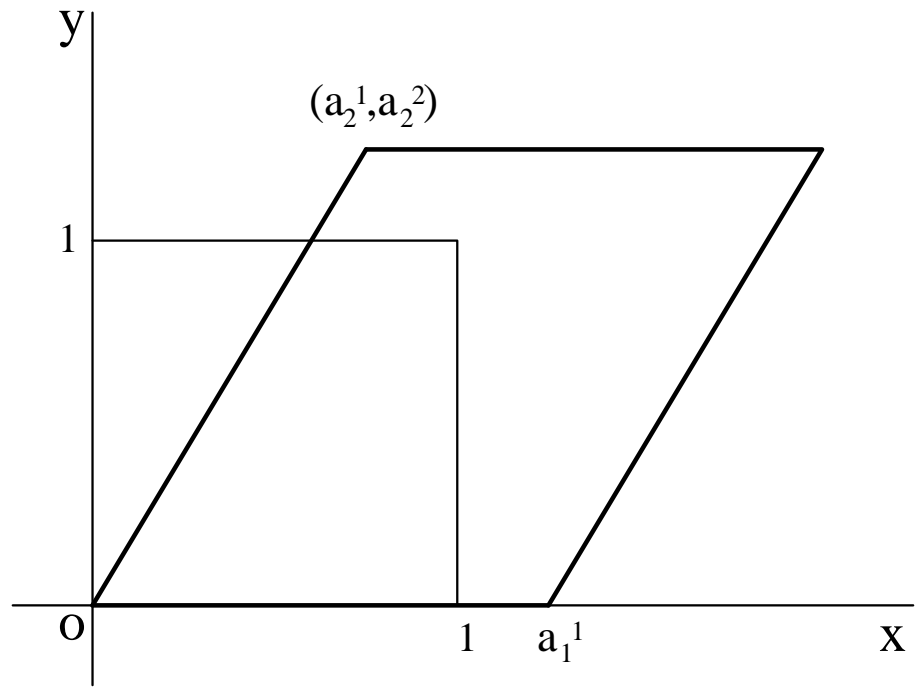

FIGURE 2 